\title{
As Estratégias de Aprendizagem Autorregulada (SRL) no Ensino EAD de Contabilidade
}

\begin{abstract}
Resumo
Os objetivos desta investigação foram identificar quais são as estratégias de aprendizagem autorregulada utilizadas por estudantes de Contabilidade do ensino Educação a Distância (EAD) e analisar como essas estratégias poderiam ser explicadas a partir do estágio (semestre) do estudante no curso. A amostra da pesquisa compreendeu discentes do curso de graduação em Ciências Contábeis na modalidade de Ensino a Distância de três instituições com polo em Salvador(BA)/Brasil. Para análise dos dados, utilizaram-se três procedimentos quantitativos visando alcançar os objetivos específicos, no qual, para identificar as estratégias de aprendizagem autorregulada foi utilizada a estatística descritiva e, para verificar como essas estratégias poderiam ser explicadas a partir do estágio (semestre) do estudante no curso, foi alcançada por meio da Análise Fatorial e o uso de testes paramétricos de comparação de médias (teste t). Os achados permitiram inferir que o perfil dos alunos da modalidade de ensino EAD, considerando a autorregulação da aprendizagem, foi de utilização significativa de estratégias de acordo ao modelo proposto pela Zimmerman e Pons (1986), em que há diferenças significativas nas médias dos discentes entre o estágio inicial e final de curso. O estudo contribui com os apontamentos dos órgãos internacionais de contabilidade sobre a aprendizagem ativa e permanente. A investigação proporciona, também, benefício à literatura sobre a modalidade de "Ensino a Distância" e à aprendizagem autorregulada por demonstrar que essa plataforma de ensino pode auxiliar na consecução da independência do aprendizado.
\end{abstract}

Palavras-chave: Estratégia de Aprendizagem; Educação a distância; Autorregulação; Órgãos Internacionais de Contabilidade; Metacognição.

\author{
Thiago Bruno de Jesus Silva \\ Mestre em Ciências Contábeis pela \\ Universidade Regional de Blumenau \\ (Furb) Professor na Universidade Federal \\ da Grande Dourados (UFGD) Comercial: \\ Rodovia Dourados, Itahum, Km 12, \\ Dourados(MS), CEP: 79.804-970 \\ E-mail: thiagobruno.silva@yahoo.com.br
}

\section{Luis Antonio Lay \\ Mestre em Ciências Contábeis pela Universidade Regional de Blumenau (FURB) Professor na Universidade do Contestado (UnC) Comercial: Av. Presidente Nereu Ramos, Jardim do Moinho, Mafra(SC), CEP: 89.300-000 E-mail: luisantoniolay@gmail.com}

\section{Nelson Hein \\ Doutor em Engenharia de Produção pela Universidade Federal de Santa Catarina (UFSC) Professor na Universidade Regional de Blumenau (Furb) Comercial: Av. Antônio da Veiga, 140, Vila Nova, Blumenau(SC), CEP: 89.010-971 \\ E-mail: hein@furb.br}

\section{Vania Tanira Biavatti}

Doutora em Ciências Sociais pela Pontifícia Universidade Católica de São Paulo (PUC-SP) Professora na Universidade Regional de Blumenau (Furb) Comercial: Av. Antônio da Veiga, 140, Vila Nova, Blumenau(SC), CEP: 89.010-971 E-mail: vania@furb.br

\section{Vinícius Costa da Silva Zonatto Pós-doutor em Ciências Contábeis pela Universidade do Vale do Rio dos Sinos (Unisinos) Professor na Universidade Regional de Blumenau (Furb) Comercial: Av. Antônio da Veiga, 140, Vila Nova, Blumenau(SC), CEP: 89.010-971 E-mail.: viniciuszonatto@gmail.com}




\section{Introdução}

A profissão contábil tem exigido da comunidade acadêmica, sobretudo das pesquisas educacionais, perspectivas para o preparo dos alunos para a vida profissional, principalmente para aprender, manter habilidades e buscar o conhecimento (Schleiger \& Dull, 2009; Martin \& Dowson, 2009). Entre as modalidades de ensino, a Educação a Distância (EAD) é cada vez mais presente como forma de levar a educação formal como mais uma opção de disseminação e compartilhamento de conhecimento por meio do seu método de aprendizado, e, ainda, é uma forma de contribuição no processo para ampliar e interiorizar a oferta de educação.

A Lei de Diretrizes e Bases da Educação Nacional (Brasil, 1996) abriu espaço na condição que a EAD se estabelecesse no cenário nacional. $\mathrm{O}$ instrumento com dimensões visando orientar o processo de credenciamento de IES e a avaliação de cursos superiores a distância, no âmbito do Sistema Nacional de Avaliação da Educação Superior (Sinaes), apresenta que o Sistema de Controle, Produção e Distribuição de Material Didático indica que os materiais utilizados necessitam promover autonomia para o aluno aprender e controlar o seu próprio desenvolvimento (Brasil, 2007). Considerando essa afirmativa, os discentes que optam pelo ensino EAD podem aprender a administrar o tempo e tornar atores de seu processo de aprendizagem. As ferramentas tecnológicas podem ajudar a melhorar a aprendizagem, incluindo a autorregulação, especialmente nas fases iniciais do ensino superior (Niemi, Harju, Vivitsou, Niittanem, Multisilta \& Kuokkanen, 2014).

Barry Zimmerman e outros pesquisadores apresentaram a perspectiva do aprendizado autorregulado ou self-regulated learning (SRL). Nessa visão, o aluno autorregulado é consciente e controla o seu processo de aprendizagem; seleciona os métodos e as estratégias que utiliza; organiza e estrutura quer o seu contexto de estudo quer o trabalho a realizar; identifica as situações em que precisa de ajuda; e adapta as estratégias de aprendizagem aos seus objetivos acadêmicos (Zimmerman \& Pons, 1986). Bertagnolli et al. (2007) encara a "Educação a Distância" como uma forma de ensino que possibilita a autoaprendizagem, com a mediação de recursos didáticos sistematicamente organizados, utilizados isoladamente ou combinados e veiculados por diversos meios de comunicação.

Bell e Akroyd (2006) concluíram que o sucesso na aprendizagem a distância correlaciona-se positivamente com a capacidade dos discentes de se autorregularem e direcionarem seus próprios esforços de aprendizagem. Ao analisar a literatura, a SRL foi essencial para o sucesso na aprendizagem a distância (Bell \& Akroyd, 2006; Lawanto, Santoso, Goodridge \& Lawanto, 2014; Puzziferro, 2008; Wang, Shannon \& Ross, 2013). Niemi et al., (2014) constataram que uma tutoria interativa baseada na Web ferramenta e IQ foi benéfica e eficaz em aumentar a autorregulação dos alunos. O software de tutoria foi especialmente útil para os discentes que não tinham estratégias estáveis de SRL ou para os alunos nas fases iniciais de estudos universitários.

Lombaerts, De Backer, Engels e Braak (2009) apontam algumas vantagens do aprendizado autônomo: permite ao aluno aprender melhor e buscar aprofundamento quanto a assunto do seu interesse, uma vez que o professor, diante das exigências curriculares institucionais e do tempo disponível, desenvolve conteúdo considerado essencial, não permitindo condições de atender às opções dos alunos; contribui para enriquecer os conhecimentos dos alunos; emancipa o aluno da dependência do professor, permitindo descobrir e formar alternativas para a construção do conhecimento; prepara o aluno para o exercício da cidadania e, assim, realiza opções conscientes na vida; e prepara para o mercado de trabalho, desenvolvendo habilidades e competências para o exercício consciente da profissão.

Em estudo, Batista, Cruz, Andrade e Brune (2014) objetivaram avaliar o nível de desempenho discente dos cursos de Ciências Contábeis no nordeste brasileiro, traçando uma comparação dos resultados obtidos pelos cursos nas modalidades "a distância" e "presencial" nos Enades de 2009 e 2012. Como resultado, os cursos na modalidade EAD promovidos pelas IES privadas obtiveram melhor desempenho que os cursos promovidos pela maioria das IES na modalidade presencial, e o resultado dos discentes "a distância" foi superior ao ensino "presencial". Assim, segundo essa pesquisa, a modalidade de "Ensino a Distância" comprovou ser uma variável significativa para o desempenho discente no Enade. 
Segundo Wang et al. (2013), a aprendizagem EAD é diferente da convencional. Atitudes autorreguladas são especialmente importantes quando se estuda nessa modalidade de ensino, visto não ter oportunidade de interagir face a face com o professor. Zimmerman e Schunk (2012) destacam que o papel dos computadores no ensino está se expandindo e oferecendo oportunidade de autorregulação. Dessa forma, considerando o exposto, este estudo busca encontrar evidências acerca do impacto que o ensino EAD exerce na postura independente e proativa, que os principais órgãos internacionais esperam dos profissionais da contabilidade. Assim, os objetivos do estudo foram identificar quais são as estratégias de aprendizagem autorregulada utilizadas por estudantes de Contabilidade do ensino EAD e analisar como essas estratégias poderiam ser explicadas a partir do estágio (semestre) do estudante no curso.

Segundo Becker (2013), apesar da defesa da aprendizagem ao longo da vida pelos órgãos internacionais de contabilidade, muitas salas de aula de Contabilidade não desenvolvem as habilidades metacognitivas (a aprendizagem ao longo da vida), no qual ainda é mais crítico nos dias atuais. Dessa forma, passados 25 anos quando a AECC recomendou a aprendizagem permanente, o desenvolvimento de habilidades metacognitivas pode parecer tarefa árdua para os professores de Contabilidade que são especialistas em sua disciplina, mas pode ser menos preparado pedagogicamente. Moos e Ringdal (2012) afirmam que o contexto educacional pode assumir papel particularmente relevante em como os alunos abordam o processo de aprendizagem e desenvolver a sua habilidade de aprendizagem.

Dessa forma, como contribuição teórica, a investigação analisa se os estudantes desenvolvem atitudes autorreguladas nessa modalidade de ensino. No que se refere à contribuição prática, os resultados podem reforçar a importância de se refletir sobre o ensino-aprendizagem desenvolvido com a intenção de armazenar conhecimentos e repensar medidas que conduzam à aprendizagem autônoma do estudante, $o$ que torna vital para o desenvolvimento de competências profissionais, que, por sua vez, requer a revisão da prática pedagógica universitária da educação contábil.

\section{Revisão de Literatura}

As mudanças das leis e procedimentos que são utilizados para a elaboração das demonstrações contábeis no Brasil é cada vez mais regular devido à internacionalização das normas contábeis. No entendimento de Becker (2011), o contador deve ter, além do conhecimento profundo, abrangente e atualizado da área, cultura humanística e domínio das Ciências Comportamentais. Deve ser um cidadão com uma visão aberta do mundo, capaz de adaptar-se facilmente a cenários de mudanças e que aceite a educação continuada como condição de vida. Paralelamente a tais mudanças, a autonomia dos discentes se configura em uma necessidade no processo formativo, seja pela modalidade de ensino "presencial" ou "a distância", com o intuito de formar profissionais com condições de aprender a aprender para se alcançar sucesso nesse ambiente propício à dinamicidade.

\subsection{Ensino EAD}

A "Educação à Distância" engloba universos de relações quando professor e aluno estão separados no tempo e/ou espaço. Tais relações são ordenadas de acordo com elementos básicos que envolvem o saber, a estrutura de programas educacionais, a interação professor-aluno e, sobretudo, a natureza e o grau de autonomia do indivíduo (Moore, 1993). 
A "Educação a Distância”, antes considerada impossível, tem, hoje, uma abertura de estratégias eficientes para a viabilidade de ensino na modalidade "virtual". Desse modo, a EAD teve um salto nas formas de ensino-aprendizagem, pois foi preciso se adaptar às necessidades das limitações que cerceiam a modalidade virtual. Desse modo, a materialização de ambientes e as metodologias educacionais inovadoras, sobretudo com o auxílio das tecnologias digitais, potencializaram a EAD, de modo que o ensino-aprendizagem fosse contemplado com uma nova política de educação (Silva, Shitsuka \& Morais, 2013) - Deve-se ter em mente, nesse caso, que essas consistem em fortes aliadas para motivar, ilustrar, apresentar e compor os conteúdos das aulas, a fim de torná-las atrativas e interativas, conforme destacam Hack e Negri (2010).

Embora tendo um importante papel na formação de milhares de pessoas, nessa modalidade de ensino não é difícil encontrar preconceito, entendida como sinonímia de curso fácil, fora das quatro paredes de uma sala de aula, sem o emprego do giz, apagador, um ou outro recurso audiovisual e a autoridade máxima do professor - preconceito advindo, possivelmente, do desconhecimento de que, além de várias fundações criadas ao redor do mundo para implantar essa modalidade de ensino, já estavam criadas universidades abertas no Reino Unido (1969), na Espanha (1972), na Austrália e Venezuela (1977), na Índia e na Dinamarca (1982) e, no Japão (1983) e na Índia novamente (1985), entre outros países (Nunes, 2009).

Dessa forma, tendo vista a complexidade para exercer a profissão contábil, dada pelos desafios e assolada por diversas alterações, seja na adição de complexidade nas normas fiscais/societárias, seja por várias funções e responsabilidade social quanto à saúde, educação das comunidades que estão inseridas e, também, com o meio ambiente, o ensino deve pleitear uma modalidade que habilite o discente a possuir condições de acompanhar as mudanças que estão intrinsecamente ligados já que serão futuros profissionais.

De acordo com o Ministério da Educação (1996) o "Ensino à Distância" é a forma de ensino que deve propiciar a autoaprendizagem, com o uso de materiais didáticos organizados e de forma a apresentar diversos suportes de informação, veiculado a diversos meios de comunicação - características desejáveis ao perfil de um aluno autorregulado, que segundo Ames (1992), se distinguem pela forma que perspectivam o seu papel no processo de aprendizagem no qual o sucesso acadêmico depende do que for construído pelo aluno. O desenvolvimento tecnológico permitiu que novos meios de comunicação fossem utilizados na mediação didático-pedagógica (Penterich, 2009). No entendimento de Anohina (2005), a tecnologia de comunicação e informação (TICs), agregada aos meios tradicionais de ensino, oferece novas oportunidades para a aquisição de conhecimento ao permitir a escolha do tempo, local, ritmo e quantidade de aprendizagem.

\subsection{Aprendizagem Autorregulada}

Na década de 70 houve discussões no campo teórico da psicologia sobre aspectos que permeiam a relação entre a memória e aprendizado, derivando o estudo da metacognição que foi, primeiramente, definida como o domínio que o indivíduo possui sobre seu próprio conhecimento. Ainda na mesma década é encorpada com o domínio dos processos e produtos cognitivos (Flavell, 1976).

A partir da metacognição é possível a autorregulação intelectual. Foram iniciadas pesquisas lideradas por Barry Zimmerman para o entendimento da autorregulação ou Self Regulated Learn (SLR). Influenciado pelo paradigma construtivista, que tem o indivíduo como agente de seu aprendizado (Arias, Lozano, Cabanach \& Pérez, 1999; Zimmerman \& Schunk, 2001; Xu, Benson, Mudrey-Camino \& Steiner, 2010), afirma-se que indivíduos autorregulados são persistentes, decididos, estratégicos e capazes de avaliar o seu progresso, diferentemente daqueles que são dependentes cognitivos, isto é pouco autorregulados. A autorregulação da aprendizagem (SRL) enfatiza a autonomia e controle do discente ao monitorar suas cognições, seus comportamentos e suas emoções orientadas para atingir as metas de aprendizagem (Cho \& Shen, 2013; Delen, Liew \& Wilson, 2014) 
Para Simons e Beukhof (1987), a autorregulação é a capacidade de o indivíduo ser 'auto-ensinante', ou seja, capaz de preparar, facilitar e regular a aprendizagem, de forma a gerar um feedback e julgamento sobre o processo. Para Costa (2001) a autorregulação é observada pelo grau de envolvimento ativo no processo de aprendizagem (metacognição, motivação e comportamental); comportamento cíclico de mudança (controle da eficácia, envolvimento e reflexão dos resultados); e dependência dos aspectos motivacionais (grau de envolvimento com relação de controles e crenças).

Zimmerman e Pons (1986) identificaram 14 estratégias presentes em estudantes autorregulados. Para esses autores, o uso dessas estratégias confere ao aluno um valioso ferramental; sua utilização está altamente correlacionada com os índices de sucesso acadêmico e com a opinião dos docentes acerca do seu grau de autorregulação em sala de aula (Tabela 1).

Tabela 1:

Estratégias de autorregulação da aprendizagem identificadas por Zimmerman e Pons (1986).

\begin{tabular}{|c|c|c|c|}
\hline & Estratégias: & Definição: & Exemplos: (Rosário, 1999) \\
\hline 1 & Auto-avaliação & $\begin{array}{l}\text { Declarações que indicam as avaliações dos } \\
\text { alunos sobre a qualidade ou progresso do seu } \\
\text { trabalho. }\end{array}$ & $\begin{array}{l}\text { "...verifiquei o meu trabalho para ter a } \\
\text { certeza que estava bem". }\end{array}$ \\
\hline 2 & $\begin{array}{l}\text { Organização e } \\
\text { transformação }\end{array}$ & $\begin{array}{l}\text { Declarações que indicam as iniciativas dos } \\
\text { alunos para reorganizarem, melhorando os } \\
\text { materiais de aprendizagem. }\end{array}$ & $\begin{array}{l}\text { "...faço sempre um esquema antes de } \\
\text { realizar os relatórios das experiências } \\
\text { de química". }\end{array}$ \\
\hline 3 & $\begin{array}{l}\text { Estabelecimento } \\
\text { de objetivos e } \\
\text { planeamento }\end{array}$ & $\begin{array}{l}\text { Declarações indicando o estabelecimento de } \\
\text { objetivos educativos: planejamento, fase no } \\
\text { tempo e conclusão de atividades relacionadas } \\
\text { com esses objetivos. }\end{array}$ & $\begin{array}{l}\text { "... começo a estudar duas semana } \\
\text { antes do teste e fico descansada". }\end{array}$ \\
\hline 4 & $\begin{array}{l}\text { Procura de } \\
\text { informação }\end{array}$ & $\begin{array}{l}\text { Declarações indicando os esforços dos alunos } \\
\text { para adquirir informações extra de fontes } \\
\text { não sociais quando enfrentaram uma tarefa } \\
\text { escolar. }\end{array}$ & $\begin{array}{l}\text { "...antes de começar um trabalho, vou à } \\
\text { biblioteca da escola recolher o máximo } \\
\text { de informações sobre o tema". }\end{array}$ \\
\hline 5 & $\begin{array}{l}\text { Tomada de } \\
\text { apontamentos }\end{array}$ & $\begin{array}{l}\text { Declaração indicando os esforços para } \\
\text { registrar eventos ou resultados. }\end{array}$ & $\begin{array}{l}\text { “...nas aulas sorvo o máximo de } \\
\text { apontamentos sobre o que o } \\
\text { professor dá". }\end{array}$ \\
\hline 6 & $\begin{array}{l}\text { Estrutura } \\
\text { ambiental }\end{array}$ & $\begin{array}{l}\text { Declarações indicando esforços para } \\
\text { selecionar ou alterar o ambiente físico } \\
\text { ou psicológico de modo a promover a } \\
\text { aprendizagem. }\end{array}$ & $\begin{array}{l}\text { “...para não me distrair, isolo-me no } \\
\text { quarto" ou “...para me concentrar no } \\
\text { que estou fazendo, desligo o som”. }\end{array}$ \\
\hline 7 & Autoconsequência & $\begin{array}{l}\text { Declarações indicando a imaginação ou a } \\
\text { concretização de recompensas ou punições } \\
\text { para sucessos ou fracassos escolares. }\end{array}$ & $\begin{array}{l}\text { "...se me der bem no teste, compro uns } \\
\text { chocolates". }\end{array}$ \\
\hline 8 & $\begin{array}{l}\text { Repetição e } \\
\text { memorização }\end{array}$ & $\begin{array}{l}\text { Declarações indicando as iniciativas e os } \\
\text { esforços dos alunos para memorizar o } \\
\text { material. }\end{array}$ & $\begin{array}{l}\text { "...na preparação de um teste de física, } \\
\text { escrevo muitas vezes a formula, até } \\
\text { saber de cor". }\end{array}$ \\
\hline $9-11$ & $\begin{array}{l}\text { Procura de ajuda } \\
\text { social }\end{array}$ & $\begin{array}{l}\text { Declarações indicando as iniciativas e os } \\
\text { esforços dos alunos para procurarem ajuda } \\
\text { dos pares (9); professores (10); e adultos (11). }\end{array}$ & $\begin{array}{l}\text { "... se tenho dificuldades no estudo } \\
\text { peço ajuda ao meu pai que é médico". }\end{array}$ \\
\hline $12-14$ & Revisão de dados & $\begin{array}{l}\text { Declarações indicando os esforços-iniciativas } \\
\text { dos alunos para relerem as notas (12); } \\
\text { teste (13); e livros de texto (14), afim de se } \\
\text { prepararem para uma aula ou exercício } \\
\text { escritos. }\end{array}$ & $\begin{array}{l}\text { "...antes dos testes revejo sempre os } \\
\text { resumos da matéria que fiz" ou "Para } \\
\text { me preparar para um teste resolvo os } \\
\text { enunciados dos que já fiz". }\end{array}$ \\
\hline
\end{tabular}

Fonte: Zimmerman e Pons (1986); Rosário, 1999, adaptado. 
O modelo de autorregulação proposto por Zimmerman e Pons (1986) é dividido em fases, componentes e processos que se reúnem com objetivo de produzir resultados na aprendizagem. A primeira fase, antecipação/preparação, tem o propósito de estabelecer os objetivos e planos estratégicos e alcançar as metas escolhidas. Nessa fase, ocorre à influência de aspectos motivacionais, de autoeficácia, de objetivos e de valorização da aprendizagem. A segunda fase, conhecida como execução e controle, tem o propósito de fazer cumprir os objetivos traçados na primeira etapa. É exigido nessa etapa automonitoração por meio do uso de estratégias de aprendizado e do controle da atenção. E, por fim, a fase de autorreflexão e autorreação que envolvem o julgamento, autoavaliação e atribuições de causa sobre os objetivos estabelecidos na primeira fase. Pode ocorrer satisfação ou insatisfação, presença de reações (derivadas da autorreflexão) e defensivas, com resistência e abandono ou satisfação e valorização pessoal. Essa fase é resultante de construtos motivacionais e cognitivos em que as três fases correspondem a um processo cíclico, pois há utilização de um feedback anterior que permite realizar alterações e contínuos aprimoramentos (Polydoro \& Azzi, 2009; Zimmerman, 2000).

Para Corno (1989), a utilização da autorregulação facilita o processo de aprendizagem, no momento em que o amadurecimento do aprendiz em criar metas, como também o ritmo próprio do seu estudo, adotando a aplicação de estratégias de monitoramento, elaboração e gerenciamento de esforços. Alunos autorregulados são geralmente caracterizados como sendo decididos, estratégicos e persistentes no seu processo de aprendizagem (Rosário, 1999).

Rosário (2001) realizou um estudo com 558 alunos secundaristas, em Portugal, e identificou que os estudantes tendem a se apropriar das estratégias de autorregulação à medida que progridem no ensino, concluindo ainda que há maior busca de informações para que se aumente a profundidade do tema estudado. Alguns estudos destacaram os benefícios da forma educacional a distância para ajudar os alunos a desenvolverem habilidades de SRL (Bell \& Akroyd, 2006; Lawanto et al., 2014; Puzziferro, 2008; Wang et al., 2013).

Valle et al. (2008) avaliaram 489 estudantes provenientes de diferentes universidades públicas da Europa, sendo a maioria deles mulheres, do primeiro ciclo (do $1^{\circ}$ ao $3^{\circ}$ ano) e identificaram três perfis de autorregulação da aprendizagem em decorrência do uso das estratégias. O primeiro corresponde ao perfil baixo, que engloba alunos que usam as estratégias de elaboração e de organização (estratégias cognitivas). O segundo, perfil moderado, envolve os alunos que gerenciam o tempo, o estudo e o esforço da autorregulação. O terceiro, perfil alto, abrange os alunos que estabelecem metas de aprendizagem e autoeficácia para aprender (estratégias motivacionais). Para eles, as estratégias cognitivas são mais preditoras da autorregulação da aprendizagem do que as estratégias motivacionais.

No Brasil, Lima Filho, Lima e Bruni (2015) pesquisaram sobre o aprendizado autorregulado em duas universidades públicas baianas, aplicando as mesmas estratégias de aprendizados apresentado por Zimmerman e Pons (1986). Assim, analisaram o aprendizado autorregulado em alunos dos cursos "presenciais" de Contabilidade, apresentando diagnósticos, dimensões e possíveis explicações, contextualizadas a partir do gênero, idade e estágio no curso. Por meio da amostra formada por 249 indivíduos, foi revelado que o gênero e a idade são fatores que influenciam o grau de autorregulação de um aluno. Mulheres e discentes mais jovens tendem a apresentar melhores níveis de aprendizagem autorregulada. Contudo, na análise do estágio do curso, os resultados não apresentaram distribuição normal, o que evidenciou a impossibilidade de perceber o aumento ou redução do grau de aprendizado autorregulado entre os respondentes.

Identificar e analisar os principais processos por meios dos quais os sujeitos (alunos) podem regular o seu aprendizado, como sujeito ativo, independente e responsável, são características fundamentais para uma adaptação adequada às exigências das constantes mutações da sociedade (Patterson \& Lee, 2010; Garner, 2009). 


\section{Trajetória Metodológica}

Os objetivos do estudo foram identificar quais são as estratégias de aprendizagem autorregulada utilizadas por estudantes de Contabilidade do ensino EAD e analisar como essas estratégias poderiam ser explicadas a partir do estágio (semestre) do estudante no curso. Assim, a pesquisa se caracteriza, quanto ao problema, como quantitativa; quanto aos objetivos, como exploratória; e, quanto aos procedimentos, como levantamento ou survey.

A amostra da pesquisa compreendeu discentes do curso de graduação em Ciências Contábeis na modalidade de "Ensino a Distância" de três instituições com polo em Salvador(BA). Vale salientar que as instituições optaram por manter o nome em sigilo. Na instituição 1, foram alcançados 81 respondentes na instituição 2 foram alcançados 94 respondentes e, na instituição 3, 76 respondentes; conforme descrito na Tabela 2.

Tabela 2:

\section{Características da amostra do estudo}

\begin{tabular}{|c|c|c|c|c|c|c|c|c|c|}
\hline IES & Instituição 1 & Instituição 2 & Instituição 3 & & & & & & Soma \\
\hline $\mathrm{Fi}$ & 81 & 94 & 76 & & & & & & 251 \\
\hline $\mathrm{Fi} \%$ & 32,27 & 37,45 & 30,28 & & & & & & 100,00 \\
\hline Gênero & Feminino & Masculino & & & & & & & \\
\hline $\mathrm{Fi}$ & 137 & 114 & & & & & & & 251 \\
\hline $\mathrm{Fi} \%$ & 54,58 & 45,42 & & & & & & & 100,00 \\
\hline Idade & Até 20 & 21 a 25 & 26 a 30 & 31 a 35 & Acim & de 36 & & & \\
\hline $\mathrm{Fi}$ & 17 & 47 & 95 & 46 & & & & & 251 \\
\hline $\mathrm{Fi} \%$ & 6,77 & 18,73 & 37,85 & 18,33 & & & & & 100,00 \\
\hline Semestre & 1 & 2 & 3 & 4 & 5 & 6 & 7 & 8 & \\
\hline $\mathrm{Fi}$ & 36 & 28 & 20 & 36 & 46 & 44 & 21 & 20 & 251 \\
\hline $\mathrm{Fi} \%$ & 14,34 & 11,16 & 7,97 & 14,34 & 18,33 & 17,53 & 8,37 & 7,97 & 100,00 \\
\hline
\end{tabular}

Fonte: elaboração própria.

A Tabela 2 destaca que foram 251 respondentes das três instituições, sendo que 54,58\% formado pelo gênero feminino. De acordo com o estudo, a faixa etária dos 26 a 30 anos representa $37,85 \%$, sendo que 6,77\% possuem até 20 anos; e já dos 31 a 35 anos e acima de 36 anos, representam 18,33\%. A amostra pode ser entendida como formada de indivíduos que já trabalham e que conciliam com a educação superior. Em relação ao semestre, $18,33 \%$ estão no $5^{\circ}$ semestre, enquanto $14,34 \%$ estão no $1^{\circ}$ semestre e $7,97 \%$ estão $8^{\circ}$ semestre, ou seja, no final do curso.

O instrumento de coleta de dados foi formado por perguntas visando à caracterização do respondente, como o gênero, idade e semestre e por perguntas com o intuito de identificar as estratégias de aprendizagem autorregulada propostas por Zimmerman e Pons (1986), conforme descritas na Tabela 3. 
Tabela 3:

Afirmações e estratégias de aprendizagem autorregulada.

\begin{tabular}{|c|c|}
\hline Afirmações & Estratégias de aprendizagem autorregulada \\
\hline $\begin{array}{l}\text { 1. Avalio o meu desempenho, vejo o que devo melhorar e } \\
\text { procuro superar as dificuldades detectadas. }\end{array}$ & 1. Autoavaliação. \\
\hline $\begin{array}{l}\text { 2. Procuro sempre elaborar um plano (esquema) antes de } \\
\text { iniciar um trabalho. }\end{array}$ & 2. Organização e transformação. \\
\hline $\begin{array}{l}\text { 3. Se tenho prova, começo a estudar o mais cedo possível, } \\
\text { para ficar descansado e tranquilo no dia. }\end{array}$ & 3. Estabelecimento de objetivos e planejamento. \\
\hline $\begin{array}{l}\text { 4. Antes de iniciar um trabalho, recorro sempre à } \\
\text { biblioteca (e outros meios de pesquisa seja físico ou } \\
\text { digital) parar separar o máximo de informação sobre o } \\
\text { tema. }\end{array}$ & 4. Procura de informação. \\
\hline $\begin{array}{l}\text { 5. Sempre procuro anotar o máximo de apontamento de } \\
\text { um texto lido ou da aula expositiva do professor. }\end{array}$ & 5. Tomada de apontamentos. \\
\hline $\begin{array}{l}\text { 6. Para ter melhor concentração, procuro sempre } \\
\text { ambiente que não proporcione distração. }\end{array}$ & 6. Estrutura ambiental. \\
\hline $\begin{array}{l}\text { 7. Quando faço uma prova, se ocorrer bem, ofereço-me } \\
\text { recompensa; caso ocorra o contrário, abro mão de algo } \\
\text { que tanto queria. }\end{array}$ & 7. Autoconsequências. \\
\hline $\begin{array}{l}\text { 8. Utilizo estratégias para memorizar o assunto (ou } \\
\text { formulas), até saber de cor do assunto a ser estudado. }\end{array}$ & 8. Repetição e memorização. \\
\hline $\begin{array}{l}\text { 9. Quando surge dificuldade e não consigo resolver } \\
\text { sozinho, busco ajuda externa (professor, colegas, outros). }\end{array}$ & $\begin{array}{l}\text { 9. Ajuda de professores; 10. Ajuda de pares próximos; e } \\
\text { 11. Ajuda de especialistas. }\end{array}$ \\
\hline $\begin{array}{l}\text { 10. Após conclusão de um trabalho acadêmico, eu o reviso } \\
\text { para ter a certeza de que ele está correto. }\end{array}$ & $\begin{array}{l}\text { 12. Revisão das anotações; 13. Revisão de testes e } 14 \text {. } \\
\text { Revisão da bibliografia. }\end{array}$ \\
\hline
\end{tabular}

Fonte: Lima Filho, Lima e Bruni (2015).

De acordo com as afirmações voltadas às respectivas estratégias de autorregulação da aprendizagem, os discentes foram convidados a basear suas respostas em sua experiência, em que deveriam atribuir uma resposta entre 1 ("nunca") e 7 ("sempre").

A hipótese deste estudo, que norteou o alcance do objetivo que foi analisar como as estratégias de aprendizagem autorregula poderiam ser explicadas a partir do estágio (semestre) do estudante no curso, está descrita na Tabela 4.

Tabela 4:

\section{Hipótese do estudo associada ao segundo objetivo}

\section{Hipótese do estudo}

$\mathrm{H} 1$ : institui que existe uma relação significativa que quanto maior for o semestre em curso de um respondente maior será o seu nível de SRL.
Fundamentação teórica

Bell e Akroyd (2006); Lawanto et al., (2014); Puzziferro, (2008); Wang et al., (2013); Niemi et al. (2014)

Fonte: elaborada pelos autores. 
A hipótese $\mathrm{H} 1$ foi amparada por conclusões de pesquisas que tiveram o mesmo objetivo. Wang et al. (2013) afirmam que a aprendizagem EAD é diferente da convencional, em que atitudes autorreguladas são especialmente importantes quando se estuda nessa modalidade de ensino, visto não ter oportunidade de interagir face a face com o professor Bell e Akroyd (2006) concluíram que o sucesso na aprendizagem "a distância" correlaciona-se positivamente com a capacidade dos discentes de se autorregularem e direcionarem seus próprios esforços de aprendizagem. Ao analisar a literatura, a SRL foi essencial para o sucesso na aprendizagem a distância (Bell \& Akroyd, 2006; Lawanto et al., 2014; Puzziferro, 2008; Wang, Shannon \& Ross, 2013). Niemi et al. (2014) constataram que uma tutoria interativa baseada na Web ferramenta e IQ foi benéfica e eficaz em aumentar a autorregulação dos alunos. O software de tutoria foi especialmente útil para os discentes que não tinham estratégias estáveis de SRL ou para os alunos nas fases iniciais de estudos universitários.

Para a análise dos dados, utilizaram-se três procedimentos quantitativos visando alcançar os objetivos específicos. Para identificar as estratégias de aprendizagem autorregulada foi utilizada a estatística descritiva com o intuito de verificar como essas estratégias poderiam ser explicadas a partir do estágio (semestre) do estudante no curso, utilizam-se a Análise Fatorial e os testes paramétricos de comparação de médias (teste $\mathrm{t}$ ).

Por fim, com os resultados encontrados para cada objetivo específico, foi possível alcançar o objetivo geral de analisar o perfil dos estudantes de Ciências Contábeis na modalidade EAD quanto às estratégias de aprendizagem autorregulada.

\section{Análise e Discussão dos Resultados}

Nesta seção serão expostos e discutidos os resultados da pesquisa. Inicialmente, para atender ao primeiro objetivo de identificar as estratégias de aprendizagem autorregulada utilizadas por estudantes de Contabilidade do ensino EAD, apresenta-se a análise descritiva dos dados. Posteriormente, foram utilizados a análise fatorial e o teste paramétrico de comparação de médias (teste t), visando à consecução do segundo objetivo de verificar como essas estratégias poderiam ser explicadas a partir do estágio (semestre) do estudante no curso.

$\mathrm{Na}$ Tabela 5 apresentam-se os resultados das estratégias de aprendizagem autorregulada utilizadas pelos discentes de contabilidade do ensino EAD. Destaca-se que as duas últimas colunas apresentam uma totalização das frequências para respostas menores que quatro, e maiores que quatro sendo um ponto médio entre 1 e 7. 
Tabela 5:

Identificar as estratégias de aprendizagem autorregulada dos discentes da EAD

\begin{tabular}{|c|c|c|c|c|c|c|c|c|c|c|c|}
\hline \multirow{2}{*}{\multicolumn{2}{|c|}{ Estratégia }} & \multicolumn{8}{|c|}{ Respostas } & \multirow{3}{*}{ Menor que 4} & \multirow{3}{*}{ Maior que 4} \\
\hline & & 1 & 2 & 3 & 4 & 5 & 6 & \multirow{2}{*}{$\begin{array}{c}7 \\
\text { Sempre }\end{array}$} & \multirow{2}{*}{ Total } & & \\
\hline & & Nunca & & & & & & & & & \\
\hline \multirow{2}{*}{ E1 } & $\mathrm{Fi}$ & 0 & 7 & 12 & 6 & 22 & 55 & 149 & 251 & 25,00 & 226,00 \\
\hline & $\mathrm{Fi} \%$ & 0,00 & 2,80 & 4,80 & 2,40 & 8,80 & 21,90 & 59,40 & 100,00 & 7,56 & 90,10 \\
\hline \multirow{2}{*}{ E2 } & $\mathrm{Fi}$ & 8 & 1 & 14 & 34 & 51 & 78 & 65 & 251 & 23,00 & 194,00 \\
\hline & $\mathrm{Fi} \%$ & 3,19 & 0,40 & 5,58 & 13,55 & 20,32 & 31,08 & 25,90 & 100,00 & 9,17 & 77,30 \\
\hline \multirow{2}{*}{ E3 } & $\mathrm{Fi}$ & 6 & 9 & 15 & 52 & 72 & 57 & 40 & 251 & 30,00 & 169,00 \\
\hline & $\mathrm{Fi} \%$ & 0,02 & 0,04 & 0,06 & 0,21 & 0,29 & 0,23 & 0,16 & 100,00 & 12,00 & 67,00 \\
\hline \multirow{2}{*}{ E4 } & $\mathrm{Fi}$ & 12 & 17 & 18 & 14 & 46 & 50 & 94 & 251 & 47,00 & 190,00 \\
\hline & $\mathrm{Fi} \%$ & 4,80 & 6,80 & 7,20 & 5,60 & 18,30 & 19,90 & 37,50 & 100,00 & 18,80 & 75,70 \\
\hline \multirow{2}{*}{ E5 } & $\mathrm{Fi}$ & 3 & 16 & 34 & 18 & 58 & 63 & 59 & 251 & 53,00 & 180,00 \\
\hline & $\mathrm{Fi} \%$ & 1,20 & 6,40 & 13,50 & 7,20 & 23,10 & 25,10 & 23,50 & 100,00 & 21,10 & 71,70 \\
\hline \multirow{2}{*}{ E6 } & $\mathrm{Fi}$ & 3 & 8 & 20 & 6 & 41 & 67 & 106 & 251 & 31,00 & 214,00 \\
\hline & $\mathrm{Fi} \%$ & 1,20 & 3,20 & 8,00 & 2,40 & 16,30 & 26,70 & 42,20 & 100,00 & 12,40 & 85,20 \\
\hline \multirow{2}{*}{ E7 } & $\mathrm{Fi}$ & 123 & 20 & 5 & 41 & 23 & 24 & 15 & 251 & 148,00 & 62,00 \\
\hline & $\mathrm{Fi} \%$ & 49,00 & 8,00 & 2,00 & 16,30 & 9,20 & 9,60 & 6,00 & 100,00 & 59,00 & 24,80 \\
\hline \multirow{2}{*}{ E8 } & $\mathrm{Fi}$ & 23 & 27 & 28 & 25 & 61 & 44 & 43 & 251 & 78,00 & 148,00 \\
\hline & $\mathrm{Fi} \%$ & 9,20 & 10,80 & 11,20 & 10,00 & 24,30 & 17,50 & 17,10 & 100,00 & 31,20 & 58,90 \\
\hline \multirow{2}{*}{ E9 } & $\mathrm{Fi}$ & 1 & 14 & 26 & 12 & 33 & 42 & 123 & 251 & 41,00 & 198,00 \\
\hline & $\mathrm{Fi} \%$ & 0,40 & 5,60 & 10,40 & 4,80 & 13,10 & 16,70 & 49,00 & 1,00 & 16,40 & 78,80 \\
\hline \multirow{2}{*}{ E10 } & $\mathrm{Fi}$ & 6 & 4 & 5 & 11 & 56 & 56 & 113 & 251 & 15,00 & 225,00 \\
\hline & $\mathrm{Fi} \%$ & 2,40 & 1,60 & 2,00 & 4,40 & 22,30 & 22,30 & 45,00 & 1,00 & 6,00 & 89,60 \\
\hline
\end{tabular}

Fonte: elaborada pelos autores.

Como resultado, os discentes da plataforma de ensino EAD utilizam as estratégias de aprendizagem autorregulada, visto que estão acima do ponto médio da escala apresentada no instrumento de coleta de dados que correspondeu ao número quatro, sendo a exceção a estratégia de autoconsequência (E7). Destaca-se que Lima Filho, Lima e Bruni (2015) encontraram o mesmo resultado para discentes de duas instituições públicas do ensino presencial. As estratégias autoavaliação (E1), revisão de anotações, revisão de testes e revisão da bibliografia (E10) e estrutura ambiental (E6) foram as mais utilizadas com soma de frequências de $90,10 \%, 89,60 \%$ e 85,205, respectivamente. As demais estratégias apresentam soma de frequência superior a $50 \%$ para as respostas maiores que quatro.

Zimmerman e Pons (1986) afirmam que o uso dessas estratégias confere ao aluno um valioso ferramental. A utilização está altamente correlacionada com os índices de sucesso acadêmico. Pavesi (2015) argumenta que, por um lado, a flexibilidade de tempo, local e recursos dos Ambientes Virtuais de Aprendizagem (AVA) representam oportunidade para muitos estudantes ingressarem no ensino superior, ou mesmo, dar continuidade em sua formação universitária. Por outro lado, esses ambientes exigem maior responsabilidade do discente, que passa a ter grande autonomia sobre o seu processo de aprendizagem. Em AVAs, os discentes podem controlar o ritmo e a sequência do processo de aprendizagem e personalizar uma série de aspectos do ambiente virtual, realizando escolhas que subsidiam o desenvolvimento de sua estrutura cognitiva (Testa \& Luciano, 2010). 
Após identificar quais são as estratégias de aprendizagem autorregulada utilizadas por estudantes de Contabilidade do ensino EAD, a partir da próxima tabela, buscou-se verificar como essas estratégias poderiam ser explicadas a partir do estágio (semestre) do estudante no curso, visando à consecução do segundo objetivo específico. Adicionalmente, verificou-se também como as estratégias poderiam ser explicadas pela idade e semestre. Para tanto, utilizou-se, primeiramente, a análise fatorial com o intuito de resumir e reduzir dados (Hair, Black, Babin, Anderson \& Tatham, 2009), conforme descrito na Tabela 6.

Tabela 6:

Análise de componente principal para as estratégias de aprendizagem (SRL)

\begin{tabular}{ccccccc}
\hline & \multicolumn{3}{c}{ Autovalor Inicial } & \multicolumn{2}{c}{ Somas extraídas dos carregamentos } \\
\cline { 2 - 7 } Componente & Total & \% da Variância & \% Acumulado & Total & \multicolumn{2}{c}{ Quadráticos } \\
\cline { 2 - 7 } & & & & & \% da Variância & \% Acumulado \\
\hline 1 & 3,255 & 32,554 & 32,554 & 3,255 & 32,554 & 32,554 \\
\hline 2 & 1,292 & 12,922 & 45,476 & 1,292 & 12,922 & 45,476 \\
\hline 3 & 1,15 & 11,501 & 56,977 & 1,15 & 11,501 & 56,977 \\
\hline 4 & 1,057 & 10,574 & 67,552 & 1,057 & 10,574 & 67,552 \\
\hline 5 & 0,808 & 8,084 & 75,635 & & & \\
\hline 6 & 0,668 & 6,678 & 82,313 & & & \\
\hline 7 & 0,549 & 5,491 & 87,804 & & & \\
\hline 8 & 0,494 & 4,935 & 92,739 & & & \\
\hline 9 & 0,403 & 4,028 & 96,768 & & & \\
\hline 10 & 0,323 & 3,232 & 100 & & & \\
\hline
\end{tabular}

Fonte: elaborada pelos autores.

Na Tabela 6, descrevem-se os componentes com os números de fatores formados para as estratégias de aprendizagem autorregulada, em que foram gerados 4 fatores, com percentual de informação para $o$ modelo de $32,55 \%$ para o primeiro fator; o segundo fator, com 45,47\%; o terceiro fator, $56,98 \%$; e $67,55 \%$ para o quarto fator. Menezes (2006) apresenta critérios de retenção em uma análise do número de fatores, em que o primeiro critério foi a utilização do teste Kaiser para retenção do número de fatores retidos, sendo considerados apenas valores superiores a 1,0; o segundo critério foi examinar a contribuição dos fatores retidos na variância do autovalor inicial, em que pode perceber que os fatores 2,3 e 4, embora tenha valor maior que 1,0, não contribui significativamente como o fator $1(3,255)$.

Utilizou-se o método de rotação oblíqua Promax para analisar os coeficientes de correlação. A rotação Promax disponibiliza duas matrizes: um padrão de matriz e uma matriz de estrutura, além da matriz fatorial, que delineiam padrões de intercorrelações entre as variáveis oblíquas (Catell, 1966). Assim, as cargas fatoriais determinam os padrões e o grau de envolvimento de cada variável com os padrões. A Pattern Matrix demonstra quais variáveis são altamente envolvidas em termos relacionadas pelas cargas fatoriais em cada padrão ou clusters, a matriz de estrutura informa a extensão da correlação das variáveis com os padrões de forma geral (Shimada, Chiusili \& Messetti, 2010). Cattell (1996) e Menezes (2006) explicam que a determinação do fator chega ao final quando se verifica a afinidade dos itens com os fatores do modelo, no qual se expurga os constructos do modelo quando o valor absoluto da carga fatorial principal do item for inferior a 0,32 , na existência de cargas fatoriais similares em dois ou mais fatores em um mesmo item, sendo a diferença entre os valores absolutos das cargas fatoriais dos itens for inferior a 0,10 e também que o fator deve ser formado por dois ou mais itens. Na Tabela 7 descrevem-se a utilização dos critérios propostos e os coeficientes de correlações com o método de Pattern Matrix. 
Tabela 7:

Análise dos coeficientes de correlações com o método de Pattern Matrix

\begin{tabular}{|c|c|c|c|c|c|c|c|c|c|c|}
\hline Dimensão & E1 & E2 & E3 & E4 & E5 & E6 & E7 & E8 & E9 & E10 \\
\hline 1 & 0,779 & 0,71 & 0,699 & 0,545 & & & & 0,411 & & \\
\hline 2 & & & & & 0,819 & 0,772 & 0,583 & 0,442 & & \\
\hline 3 & & & & 0,401 & & & 0,565 & 0,394 & 0,838 & \\
\hline 4 & & & & & & & & $-0,396$ & & 0,828 \\
\hline
\end{tabular}

Fonte: elaborada pelos autores.

A partir dos critérios, o item E8 (repetição e memorização), na dimensão 4, foi excluído por apresentar valor inferior que 0,32 , os itens E9 (ajuda externa) e E10 (revisões) foram excluídos seguindo o terceiro critério, em que um fator deve ser formado por dois ou mais itens. Restaram duas dimensões: a primeira, formada por E1 (auto-avaliação), E2 (organização e transformação), E3 (estabelecimento de objetivos e planejamento), E4 (procura de informação), a segunda formada por E5 (tomada de apontamentos), E6 (estrutura ambiental), E7 (auto-consequência) e E8 (repetição e memorização). Dessa forma, as dimensões foram intituladas de acordo com a afirmação de Corno (1989), cujaautorregulação facilita o processo de aprendizagem no momento em que o amadurecimento do aprendiz em criar metas tem ritmo próprio do seu estudo, ao aplicar estratégias de monitoramento, elaboração e gerenciamento de esforços. Assim, a primeira dimensão foi intitulada Planejamento SRL e a segunda dimensão de Execução e Controle SRL.

Na Tabela 8, têm-se as verificações de dimensionalidade, confiabilidade e convergência da análise fatorial.

Tabela 8:

Teste de Validação dos Fatores das Estratégias de Aprendizagem Autorregulada (SRL)

\begin{tabular}{|c|c|c|}
\hline $\begin{array}{l}\text { Etapas e técnicas ou } \\
\text { Estatísticas }\end{array}$ & Regras para validação & Fatores \\
\hline \multicolumn{3}{|c|}{ Dimensionalidade } \\
\hline Componentes principais & $\begin{array}{c}\text { Apenas um autovalor deve existir na } \\
\text { escala }\end{array}$ & $\begin{array}{l}\text { Dois autovalores na escala (autovalor igual } \\
10,574 \text {, explicando } 67,552 \% \text { da variância). }\end{array}$ \\
\hline \multirow{2}{*}{ Índice KMO } & Maior que 0,7: Desejável & \multirow{2}{*}{ KMO igual a 0,711, desejável. } \\
\hline & Menor que 0,5: Inaceitável & \\
\hline Teste de esferidade de Bartlett & & $\begin{array}{c}\text { Qui-quadrado igual a 572,406 com nível de } \\
\text { significância igual a 0,000. }\end{array}$ \\
\hline \multicolumn{3}{|c|}{ Confiabilidade } \\
\hline Alfa de Cronbach & & Alfa de Cronbach = 0,762. \\
\hline \multicolumn{3}{|c|}{ Convergência } \\
\hline Coeficiente de Pearson & Coeficientes de Pearson $>0$ & $\begin{array}{c}\text { Todos os coeficientes de Pearson foram } \\
\text { positivos e significativos. }\end{array}$ \\
\hline
\end{tabular}

Fonte: elaboração própria.

Na Tabela 8, o valor do teste KMO foi considerado desejável (0,711). O teste de Esferidade de Bartlett foi de baixo nível de significância, e o Alfa de Conbrach $(0,762)$ também foi considerado desejável. Na Tabela 5, apresenta-se a estatística descritiva das dimensões intituladas Planejamento SRL e Execução e Controle SRL. 
Tabela 8:

\section{Estatística descritiva das dimensões}

\begin{tabular}{cccccc}
\hline Dimensão & N & Mínimo & Máximo & Média & Desvio-Padrão \\
\hline Planejamento SRL & 251 & 1,00 & 7,00 & 4,98 & 1,98616 \\
\hline Execução e Controle SRL & 251 & 1,00 & 7,00 & 6,54 & 0,78756 \\
\hline
\end{tabular}

Fonte: elaborada pelos autores.

Na dimensão Planejamento SRL, a média foi de 4,98, com desvio-padrão que demonstra a dispersão dos dados de 1,98616. Já na dimensão Execução e Controle SRL, a média foi de 6,54, com dispersão dos dados de 0,78756 . Por meio dos resultados, pode-se inferir que os discentes usam estratégias de aprendizagem autorregulada de forma relativamente alta, visto que as médias foram superiores ao ponto médio, destacando-se a média da dimensão Execução e Controle SRL que se aproximou do valor máximo $(7,00)$.

Por meio das Tabelas 8 e 9, tem-se a consecução do segundo objetivo específico, que foi verificar como essas estratégias poderiam ser explicadas a partir do estágio (semestre) do estudante no curso, por meio dos Testes de Igualdade de Média (teste t): primeiramente, na Tabela 6, com os resultados para a dimensão Planejamento SRL, e, em seguida, os resultados para a dimensão Controle e Execução SRL. Conforme mencionado anteriormente, também se analisou como as estratégias de aprendizagem autorregulada poderiam ser explicadas a partir do gênero e idade dos discentes, seguindo Valle et al. (2008), Zhao, Chen e Panda (2014), que encontraram diferença entre gênero na "Educação a Distância", e Lima Filho, Lima e Bruni (2015) concluíram que o gênero e a idade são fatores que influenciam o grau de autorregulação de um aluno no contexto da educação "presencial".

Destaca-se que a amostra foi segregada em dois grupos, de acordo com o estágio do curso (até o quinto semestre e a partir do sexto semestre), o gênero (masculino e feminino) e idade (até 24 anos e acima de 24 anos).

Tabela 8:

Testes de Igualdade de Média Dimensão Planejamento SRL

\begin{tabular}{|c|c|c|c|c|c|c|c|c|c|}
\hline & \multirow{2}{*}{$\mathbf{N}$} & \multirow{2}{*}{ Média } & \multirow{2}{*}{$\begin{array}{l}\text { Desvio } \\
\text { Padrão }\end{array}$} & \multirow{2}{*}{$\begin{array}{c}\text { Erro } \\
\text { padrão } \\
\text { da } \\
\text { média }\end{array}$} & \multicolumn{2}{|c|}{$\begin{array}{l}\text { Teste de } \\
\text { Levene }\end{array}$} & \multicolumn{3}{|c|}{ T teste } \\
\hline & & & & & $\mathrm{F}$ & Sig. & $\mathrm{t}$ & $\begin{array}{l}\text { Graus de } \\
\text { liberdade }\end{array}$ & Sig. bi \\
\hline \multicolumn{10}{|l|}{ Estágio no curso } \\
\hline Até o $5^{\circ}$ semestre & 120 & 5,2901 & 1,69836 & 0,14839 & \multirow{2}{*}{2,304} & \multirow{2}{*}{0,253} & 3,154 & 249 & 0,002 \\
\hline A partir do $6^{\circ}$ semestre & 131 & 5,9417 & 1,56267 & 0,14265 & & & 3,166 & 248,994 & 0,002 \\
\hline \multicolumn{10}{|l|}{ Gênero } \\
\hline Feminino & 137 & 5,8029 & 1,58039 & 0,13502 & \multirow{2}{*}{4,046} & \multirow{2}{*}{0,045} & $-2,098$ & 249 & 0,037 \\
\hline Masculino & 114 & 5,3596 & 1,73525 & 0,16252 & & & $-2,116$ & 231,276 & 0,035 \\
\hline \multicolumn{10}{|l|}{ Idade } \\
\hline$>=24$ anos & 187 & 5,6578 & 1,56936 & 0,11476 & \multirow{2}{*}{7,370} & \multirow{2}{*}{0,007} & $-0,914$ & 249 & 0,362 \\
\hline$<24$ anos & 64 & 5,4375 & 1,91796 & 0,23975 & & & $-0,829$ & 93,517 & 0,409 \\
\hline
\end{tabular}

Fonte: elaborada pelos autores. 
Os resultados apresentados na Tabela 8 indicam uma estatística t com nível de significância dos resultados menores que 0,05 para o estágio do curso e o gênero. Assim, rejeita-se a hipótese nula de igualdade. Dessa forma, torna-se possível concluir um aumento do uso de estratégias de aprendizagem autorregulada entre os discentes em estágio inicial e no final do curso. Também pode-se inferir que há diferenças significativas na aprendizagem autorregulada, considerando os gêneros, no qual o gênero feminino utiliza maior número de estratégias de aprendizagem autorregulada. No entanto, não foi possível perceber diferenças significativas quando se considera a idade dos discentes.

Por último, na Tabela 9, apresentam-se os resultados para a dimensão Controle e Execução SRL para o semestre, gênero e idade.

Tabela 9:

Testes de Igualdade de Média Dimensão Controle e Execução SRL

\begin{tabular}{|c|c|c|c|c|c|c|c|c|c|}
\hline & \multirow{2}{*}{$\mathbf{N}$} & \multirow{2}{*}{ Média } & \multirow{2}{*}{$\begin{array}{l}\text { Desvio } \\
\text { Padrão }\end{array}$} & \multirow{2}{*}{$\begin{array}{l}\text { Erro padrão } \\
\text { da média }\end{array}$} & \multicolumn{2}{|c|}{$\begin{array}{l}\text { Teste de } \\
\text { Levene }\end{array}$} & \multicolumn{3}{|c|}{$T$ teste } \\
\hline & & & & & $F$ & Sig & $\mathrm{t}$ & $\begin{array}{l}\text { Graus de } \\
\text { liberdade }\end{array}$ & Sig. bi \\
\hline \multicolumn{10}{|l|}{ Estágio no curso } \\
\hline Até o $5^{\circ}$ semestre & 120 & 6,3958 & 0,86213 & 0,0787 & \multirow{2}{*}{11,538} & \multirow{2}{*}{0,001} & $-2,851$ & 249 & 0,005 \\
\hline A partir do $6^{\circ}$ semestre & 131 & 6,6756 & 0,68899 & 0,0602 & & & $-2,823$ & 227,647 & 0,005 \\
\hline \multicolumn{10}{|l|}{ Gênero } \\
\hline Feminino & 137 & 6,54 & 0,77876 & 0,80135 & \multirow{2}{*}{0,029} & \multirow{2}{*}{0,865} & 0,163 & 249 & 0,87 \\
\hline Masculino & 114 & 6,5329 & 0,80135 & 0,07505 & & & $-0,164$ & 238,176 & 0,87 \\
\hline \multicolumn{10}{|l|}{ Idade } \\
\hline$\geq 24$ anos & 187 & 6,5722 & 0,81204 & 0,5938 & \multirow{2}{*}{2,352} & \multirow{2}{*}{0,126} & $-1,044$ & 249 & 0,297 \\
\hline$<24$ anos & 64 & 6,4531 & 70973 & 0,8872 & & & $-1,115$ & 123,688 & 2,677 \\
\hline
\end{tabular}

Fonte: elaborada pelos autores.

Para uma estatística t com nível de significância dos resultados menores que 0,05 , apenas para o estágio do curso, rejeita-se a hipótese nula de igualdade. Assim, pode-se inferir que os discentes aumentam o grau de utilização de estratégia de aprendizagem autorregulada durante o avanço na escala educativa. No entanto, não foi possível perceber diferenças significativas quando se consideram a idade e o gênero do discentes para a dimensão Execução e Controle SRL.

As conclusões das Tabelas 6 e 7 auxiliam inferir que há diferenças significativas nas médias dos discentes entre o estágio inicial e final de curso. Dessa forma, aceita-se a hipótese que instituiu que existe uma relação significativa que quanto maior for o semestre em curso de um respondente maior será o seu nível de SRL. Os achados do estudo são corroborados pelos estudos do Bell e Akroyd (2006); Lawanto et al., (2014); Puzziferro, (2008); Wang et al., (2013); Niemi et al. (2014). Assim, pode-se afirmar que a aprendizagem EAD é diferente da convencional. Atitudes autorreguladas são especialmente importantes quando se estuda nessa modalidade de ensino, visto não ter oportunidade de interagir face a face com o professor, conforme Wang et al., (2013).

Quanto ao gênero, apenas para a dimensão Planejamento SRL foi possível inferir que há diferença estatística de médias, no qual as mulheres utilizam mais estratégias que os homens, corroborando parcialmente os estudos do Valle et al. (2008), Zhao et al. (2014). Vale mencionar que o estudo Lima Filho, Lima e Bruni (2015) encontrou o mesmo achado, considerando o ensino presencial. 


\subsection{Discussão dos Resultados}

Os resultados demonstram que a "Educação a Distância" disponibiliza características voltadas à autonomia e ao controle do discente, e a monitorar suas cognições, comportamentos e emoções orientadas a atingir a meta de aprendizagem (Delen, Liew \& Wilson, 2014). Os estudantes com uma aprendizagem autorregulada bem-sucedida estabelecem os objetivos diretos da sua aprendizagem, monitoram, regulam e controlam a própria cognição, a motivação e o comportamento com o propósito de realizar/atingir objetivos traçados. Dessa forma, uma aprendizagem autorregulada se concretiza na medida em que os alunos enfrentam os problemas, aplicam as estratégias, monitorizam a sua realização e interpretam os resultados dos seus esforços de uma forma autônoma e centrada na tarefa (Zimmerman \& Schunk, 2011).

Nos achados, quanto às estratégias de aprendizagem autorregulada utilizadas pelos discentes de Contabilidade do ensino EAD, as estratégias autoavaliação (E1), revisão de anotações, revisão de testes, revisão da bibliografia (E10) e estrutura ambiental (E6) foram as mais utilizadas com soma de frequências de $90,10 \%, 89,60 \%$ e 85,20 , respectivamente. As demais estratégias apresentam soma de frequências superior a $50 \%$ para as respostas maiores que quatro. Quando comparado com o estudo do Lima Filho, Lima e Bruni (2015), no contexto presencial de duas universidades baianas, os resultados foram superiores, visto que nas mesmas estratégias o grau de utilização foi maior na plataforma de "Ensino a Distância".

Quando explicada as estratégias de aprendizagem autorregulada por meio do estágio do curso e, adicionalmente, o gênero e a idade. Os resultados permitiram afirmar que as médias são significativamente diferentes aos discentes no estágio inicial e final do curso, tanto para a dimensão Planejamento SRL e Controle e Execução SRL, conforme discutida no final do tópico anterior. Considerando os achados, os discentes que optam pelo ensino EAD podem aprender a administrar o tempo e tornar atores de seu processo de aprendizagem. Korkmaz e Kaya (2012) afirmam que se torna ponto-chave para o sucesso, no contexto educacional; o desenvolvimento de estratégias cognitivas e metacognitivas que levem o aluno a aprender a aprender. As ferramentas tecnológicas podem ajudar a melhorar a aprendizagem, incluindo a autorregulação, especialmente nos estágios iniciais do ensino superior (Niemi et al., 2014).

O Accounting Education Change Commission. Objectives of education for accountants (AECC, 1990), o American Institute of Certified Public Accountants Institute of Management Accountants (AICPA, 2000) e o Institute of Management Accountants (IMA, 2008) apontam para a necessidade de uma formação em sala de aula que conduza os estudantes (de Contabilidade) a adquirirem atributos e habilidades de aprendizagem permanente. Smith (2001) afirma que os profissionais da contabilidade devem possuir postura crítica e assumir o papel de aprendizes ao longo de sua trajetória profissional. Os resultados do ensino EAD são convergentes as perspectivas de aprendizagem permanente e do papel ativo, de acordo ao uso das estratégias de aprendizagem autorregulada (SRL).

Para Becker (2013), a autorregulação da aprendizagem possui impacto positivo, sobretudo aos graduados ao entrarem no ambiente dinâmico de trabalho na contabilidade dos dias atuais. Howieson, Hancock, Segal, Kavanagh, Tempone e Kent (2014) explicam que a mudança é uma constante nas organizações modernas. Dessa forma, o aconselhamento nas empresas exige maior capacidade de lidar com a incerteza e habilidades na resolução de problemas. Assim, o desenvolvimento profissional, como um processo de educação, deve enfatizar o "aprender a aprender".

Nessa esteira, o desenvolvimento e o aumento de utilização de estratégias por discentes no estágio final do curso pode explicar o resultado encontrado pelo estudo recente do Batista et al., (2014), no qual encontrou que a modalidade de "Ensino a Distância" comprovou ser uma variável significativa para o desempenho discente no Enade. Contudo, Becker (2011) afirma que a autonomia configura uma necessidade do processo formativo, seja pela modalidade de ensino "presencial" ou "a distância", e que contribui para a formação de profissionais com condições de aprender a aprender para se alcançar sucesso neste ambiente dinâmico da profissão contábil. 
Em síntese, pode-se inferir que o perfil dos alunos da modalidade de ensino EAD, quanto à autorregulação da aprendizagem, foi de uso significativo de estratégias de acordo ao modelo proposto pela Zimmerman e Pons (1986). Destacam-se que as estratégias autoavaliação (E1), revisão de anotações, revisão de testes e revisão da bibliografia (E10) e estrutura ambiental (E6) foram as mais utilizadas, com soma de frequências de $90,10 \%, 89,60 \%$ e 85,20 , respectivamente, bem como diferenças significativas aos alunos de estágio inicial para o estágio final do curso.

\section{Considerações Finais}

Os objetivos deste estudo foram identificar quais são as estratégias de aprendizagem autorregulada utilizadas por estudantes de Contabilidade do ensino EAD e analisar como essas estratégias poderiam ser explicadas a partir do estágio (semestre) do estudante no curso.

A amostra da pesquisa compreendeu discentes do curso de graduação em Ciências Contábeis na modalidade de "Ensino a Distância" de três instituições com polo em Salvador(BA). O instrumento de coleta de dados foi formado por perguntas visando à caracterização do respondente, como o gênero, idade e semestre e por perguntas com o intuito de identificar as estratégias de aprendizagem autorregulada propostas por Zimmerman e Pons (1986). Para a análise dos dados, utilizaram-se três procedimentos quantitativos visando alcançar os objetivos específicos, no qual, para identificar as estratégias de aprendizagem autorregulada, foi utilizada a estatística descritiva; e, para verificar como essas estratégias poderiam ser explicadas a partir do estágio (semestre) do estudante no curso foram utilizados a Análise Fatorial e os testes paramétricos de comparação de médias (teste $\mathrm{t}$ ).

Como resultado, as estratégias autoavaliação (E1), revisão de anotações, revisão de testes, revisão da bibliografia (E10) e estrutura ambiental (E6) foram as mais utilizadas, com soma de frequências de $90,10 \%, 89,60 \%$ e $85,20 \%$, respectivamente. As demais estratégias apresentam soma de frequências superior a $50 \%$ para as respostas maiores que quatro; também mostrou que há diferenças significativas nas médias dos discentes entre o estágio inicial e final de curso. Assim, pode-se inferir que o perfil dos alunos da modalidade de ensino EAD, considerando a autorregulação da aprendizagem, foi de utilização significativa de estratégias, de acordo ao modelo proposto pela Zimmerman e Pons (1986).

Adicionalmente, verificou-se também como as estratégias poderiam ser explicadas pela idade e semestre. Quanto ao gênero, apenas para a dimensão Planejamento SRL foi possível inferir parcialmente que há diferença estatística de médias, no qual as mulheres utilizam mais estratégias que os homens; e na idade não houve diferença de médias para alunos até 24 anos e acima de 24 anos.

Os resultados sugerem a existência de perfis diferentes, visto ter diferenças significativas entre as estratégias utilizadas ao longo do curso. Dessa forma, os estudos futuros podem investigar os diferentes tipos de perfis ao considerar o gênero e a idade, por exemplo.

O estudo contribui com as discussões dos órgãos internacionais de Contabilidade sobre a aprendizagem ativa, da aprendizagem permanente, visto que os discentes, futuros profissionais de contabilidade, necessitam desenvolver tais habilidades. O estudo também disponibiliza a literatura sobre a modalidade de "Ensino a Distância" e a aprendizagem autorregulada por demonstrar que esta plataforma de ensino contribui para a consecução de independência do aprendizado por meio das estratégias de aprendizagem que conduz a autorregulação. 


\section{Referências}

AECC - Accounting Education Change Commission (1990, Fall). Position statement no. one: objectives of education for accountants. Issues In Accounting Education, 5, pp.307-312.

AICPA - American Institute of Certified Public Accountants (2000). The AICPA core competency framework for entry into the accounting profession.

Ames, C. (1992). Achievement goals and the classroom motivational climate.Student perceptions in the classroom, pp. 327-348.

Anohina, A. (2005). Analysis of the terminology used in the field of virtual learning, Educational Technology \& Society, 8(3), pp. 91-102.

Arias, A. V., Lozano, A.B., Cabanach, R.G., Pérez, J.C.N. (1999). Las estratégias de aprendizaje: revisión teorica y conceptual. Revista Latinoamericana de Psicologia, 31 (3), pp. 425-461.

Batista, A. B., Cruz, N. V. S., Andrade, C. M., \& Bruni, A. L. (2014). Desempenho discente nos Enades 2009 e 2012 do curso de Ciências Contábeis do Nordeste Brasileiro: uma análise comparativa entre o ensino à distância e o ensino presencial. In Anais do Congresso Brasileiro de Custos, Natal, RN, Brasil, 21.

Becker, L. L. (2011). Self-Regulated Learning in an Introductory Undergraduate Accounting Course. Dissertação de doutorado em Educação em Liderança, 179 f., Universidade Estadual do Leste de Tennessee, EUA.

Becker, L. L. (2013). Self-Regulated Learning Interventions in the Introductory Accounting Course: An Empirical Study. Issues in Accounting Education, 28(3), pp. 435-460. doi: http://sci-hub.cc/10.2308/ iace-50444

Bell, P. D. \& Akroyd, D. (2006) Can factors related to self-regulated learning predict learning achievement in undergraduate asynchronous Web-based courses. International Journal of Instructional Technology and Distance Learning. 3(10), pp. 5-16.

Bertagnolli, S. D., Silveira, S. R. D., Barcelos, L. A., \& Nunes, E. (2007). O Uso de Atividades Semipresenciais em Cursos Presenciais como Forma de Qualificação da Educação Superior: o caso do UniRitter. RENOTE-Revista Novas Tecnologias na Educação, 5(1).

Cattell, R. B. (1966). The scree test for the number of factors. Multivariate behavioral research, 1(2), pp. 245-276. doi: 10.1207/s15327906mbr0102_10

Cho, M. H., \& Shen, D. (2013). Self-regulation in online learning. Distance education, 34(3), pp. 290-301. doi: 10.1080/01587919.2013.835770

Corno, L. (1989). Self-regulated learning: A volitional analysis. In Self-regulated learning and academic achievement (pp. 111-141). Springer New York. doi: 10.1007/978-1-4612-3618-4_5

Costa, J. J. M. (2001). Auto-regulação da aprendizagem: para uma caracterização multidimensional do desempenho académico. Dissertação de Doutoramento em Psicologia, especialização em Psicologia da Motivação e da Personalidade, não publicada. Faculdade de Psicologia e de Ciências da Educação da Universidade de Coimbra.

Delen, E., Liew, J., \& Willson, V. (2014). Effects of interactivity and instructional scaffolding on learning: Self-regulation in online video-based environments. Computers \& Education, 78, pp. 312-320. doi: 10.1016/j.compedu.2014.06.018

Flavell, J. H. (1976). Metacognitive aspects of problem solving. In L. B. Resnick (Ed.), The nature of intelligence (pp. 231-235). Hillsdale, NJ: Erlbaum. 
Garner, J. K. (2009). Conceptualizing the relations between executive functions and self-regulated learning. The Journal of Psychology, 143(4), pp. 405-426. doi: 10.3200/JRLP.143.4.405-426

Hack, J. R., \& Negri, F. (2010). Escola e tecnologia: a capacitação docente como referencial para a mudança. Ciências \& Cognição, 15(1), pp. 89.

Hair, J. F., Black, W. C., Babin, B. J., Anderson, R. E., \& Tatham, R. L. (2009). Análise multivariada de dados. Bookman Editora.

Howieson, B., Hancock, P., Segal, N., Kavanagh, M., Tempone, I., \& Kent, J. (2014). Who should teach what? Australian perceptions of the roles of universities and practice in the education of professional accountants.Journal of Accounting Education, 32(3), pp. 259-275. doi: 10.1016/j.jaccedu.2014.05.001

Institute Of Management Accountants. (2008) Cost Management Update, IMA, Montvale, NJ.

Jones, M. H., Alexander, J. M., \& Estell, D. B. (2010). Homophily among peer groups members' perceived self-regulated learning. The Journal of Experimental Education, 78(3), pp. 378-394. doi: $10.1080 / 00220970903548020$

Korkmaz, O., \& Kaya, S. (2012). Adapting Online Self-Regulated Learning Scale into Turkish. Turkish Online Journal of Distance Education, 13(1), pp. 52-67.

Kumar, V., \& Stracke, E. (2007). An analysis of written feedback on a PhD thesis. Teaching in Higher Education, 12(4), pp. 461-470. doi: 10.1080/13562510701415433

Lawanto, O., Santoso, H. B., Goodridge, W., \& Lawanto, K. N. (2014). Task Value, Self-Regulated Learning, and Performance in a Web-Intensive Undergraduate Engineering Course: How Are They Related? Journal of Online Learning and Teaching, 10(1), pp. 97.

Lei no 9.394, de 20 de dezembro de 1996. Dispõe sobre a Lei de Diretrizes e Bases da Educação Nacional. Diário Oficial da União, Brasília, DF: Ministério da Educação.

Lima Filho, R. N., de Lima, G. A. S. F., \& Bruni, A. L. (2015). Self-regulated learning in accounting: diagnosis, dimensions and explanations. Brazilian Business Review, 12(1), pp. 36-54.

Lombaerts, K., De Backer, F., Engels, N., Van Braak, J., \& Athanasou, J. (2009). Development of the self-regulated learning teacher belief scale.European Journal of Psychology of Education, 24(1), pp. 7996. doi: $10.1007 / \mathrm{BF} 03173476$

Martin, A. J., \& Dowson, M. (2009). Interpersonal relationships, motivation, engagement, and achievement: Yields for theory, current issues, and educational practice. Review of educational research, 79(1), pp. 327-365. doi: 10.3102/0034654308325583

Ministério da Educação e Cultura. (2007) Censo da educação superior - 2007. Brasília: Inep/Deed.

Ministério da Educação e Cultura. (2012) Instrumentos de avaliação de cursos de graduação presencial e a distância. Brasília: MEC.

Menezes, I. G. (2006). Escalas de Intenções Comportamentais de Comprometimento Organizacional (EICCO): concepção, desenvolvimento, validação e padronização. Dissertação de Mestrado em Psicologia, Universidade Federal da Bahia, Salvador, BA, Brasil.

Moore, M. G. (1993). Is teaching like flying? A total systems view of distance education. American Journal of Distance Education, 7(1), pp. 1-10.

Moos, D. C., \& Ringdal, A. (2012). Self-regulated learning in the classroom: A literature review on the teacher's role. Education Research International.

Niemi, H., Harju, V., Vivitsou, M., Viitanen, K., Multisilta, J., \& Kuokkanen, A. (2014). Digital Storytelling for 21 st-Century Skills in Virtual Learning Environments. Creative Education, 2014. doi: 10.4236/ ce.2014.59078 
Nunes, I. B. (2009). A história da EAD no mundo. Educação a distância: o estado da arte. São Paulo: Pearson Education do Brasil, pp. 2-8.

Patterson, J. T., \& Lee, T. D. (2010). Self-regulated frequency of augmented information in skill learning. Canadian Journal of Experimental Psychology/Revue canadienne de psychologie expérimentale, 64(1), pp. 33. doi: 10.1037/a0016269

Pavesi, M. A. (2015). Análise da aprendizagem autorregulada de alunos de cursos a distância em função das áreas de conhecimento, faixa etária e sexo. Dissertação de Mestrado em Educação, Universidade Estadual de Londrina, Centro de Educação, Comunicação e Artes, Programa de Pós-Graduação em Educação, Londrina, PR, Brasil.

Penterich, E. (2009). Competências organizacionais para a oferta da educação a distância no ensino superior: um estudo descritivo-exploratório de IES brasileiras credenciadas pelo MEC. Dissertação de doutorado,Universidade de São Paulo, São Paulo, SP, Brasil.

Polydoro, S. A. J., \& Azzi, R. G. (2009). Autorregulação da aprendizagem na perspectiva da teoria sociocognitiva: introduzindo modelos de investigação e intervenção. Psicologia da Educação, (29), pp. 75-94.

Puzziferro, M. (2008). Online technologies self-efficacy and self-regulated learning as predictors of final grade and satisfaction in college-level online courses. The Amer. Jrnl. of Distance Education, 22(2), pp. 72-89. doi: 10.1080/08923640802039024

Rosário, P. (1999). Variáveis cognitivo-motivacionais na aprendizagem: As abordagens ao estudo em alunos do Ensino Secundário. Tese de doutorado, Universidade do Minho, Braga, Portugal.

Rosário, P. (2001). Diferenças processuais na aprendizagem: avaliação alternativa das estratégias de autoregulação da aprendizagem. Psicologia, Educação e Cultura, 5(1), 87-102.

Schleifer, L. L., \& Dull, R. B. (2009). Metacognition and performance in the accounting classroom. Issues in Accounting Education, 24(3), pp. 339-367. doi: 10.2308/iace.2009.24.3.339

Shimada, A. T., Chiusoli, C. L., \& Messetti, A. V. L. (2010). Análise fatorial: avaliação de estabelecimentos alimentícios. Anais Seminários em Administração, São Paulo, SP, 13. doi: 10.1006/mare.2002.0183

Silva, P. C. D., Shitsuka, R., \& Morais, G. (2013). Estratégias de Ensino/Aprendizagem em Ambientes Virtuais: Estudo Comparativo do Ensino de Língua Estrangeira no Sistema EaD e Presencial. Revista Brasileira de Aprendizagem Aberta e a Distância., São Paulo, 12.

Simons, P. R. J., \& Beukhof, G. (1987). The regulation of learning. Regulation of learning, 3-11.

Smith, P. A. (2001). Understanding self-regulated learning and its implications for accounting educators and researchers. Issues in Accounting Education,16(4), pp. 663-700. doi: http://sci-hub.cc/10.2308/ iace.2001.16.4.663

Testa, M. G., \& Luciano, E. M. (2010). A influência da autorregulação dos recursos de aprendizagem na efetividade dos cursos desenvolvidos em ambientes virtuais de prendizagem na internet. REAd-Revista Eletrônica de Administração, 16(2), pp. 481-513.

Valle, A., Núñez, J. C., Cabanach, R. G., González-Pienda, J. A., Rodríguez, S., Rosário, P., \& Muñoz-Cadavid, M. A. (2008). Self-regulated profiles and academic achievement. Psicothema, 20(4), pp. 724-731.

Wang, C. H., Shannon, D. M., \& Ross, M. E. (2013). Students' characteristics, self-regulated learning, technology self-efficacy, and course outcomes in online learning. Distance Education, 34(3), pp. 302323. doi: $10.1080 / 01587919.2013 .835779$

Xu, M., Benson, S. N. K., Mudrey-Camino, R., \& Steiner, R. P. (2010). The relationship between parental involvement, self-regulated learning, and reading achievement of fifth graders: A path analysis using the ECLS-K database. Social Psychology of Education, 13(2), pp. 237-269. doi: 10.1007/ s11218-009-9104-4 
Zhao, H., Chen, L., \& Panda, S. (2014). Self-regulated learning ability of Chinese distance learners. British Journal of Educational Technology, 45(5), pp. 941-958. doi: 10.1111/bjet.12118

Zimmerman, B. J., \& Pons, M. M. (1986). Development of a structured interview for assessing student use of self-regulated learning strategies.American educational research journal, 23(4), pp. 614-628. doi: $10.1037 / 10213-000$

(2000). Self-efficacy: An essential motive to learn. Contemporary educational psycho$\log y, 25(1), 82-91$. doi:10.1006/ceps.1999.1016

, \& Schunk, D. H. (Eds.). (2001). Self-regulated learning and academic achievement: Theoretical perspectives. Routledge..

\& Schunk, D. H. (Eds.). (2011). Handbook of self-regulation of learning and performance. Taylor \& Francis.

\& Schunk, D. H. (Eds.). (2012). Self-regulated learning and academic achievement: Theory, research, and practice. Springer Science \& Business Media. 